\title{
The Correlation Between Mutant p53 Protein Expression and Cell Atypia in Early Differentiated Gastric Adenocarcinoma
}

\author{
Ming Tang ${ }^{1,2}$ \\ Peng-Jie Liu $^{3}$ \\ Bing Yue ${ }^{2}$ \\ Xuan-Tao Yang' \\ Guang-Yong Chen ${ }^{2}$ \\ 'Department of Pathology, The First \\ People's Hospital of Yunnan Province, \\ The Affiliated Hospital of Kunming \\ University of Science and Technology, \\ Kunming, 650032, People's Republic of \\ China; ${ }^{2}$ Department of Pathology, Beijing \\ Friendship Hospital, Capital Medical \\ University, Beijing, 100050, People's \\ Republic of China; ${ }^{3}$ Department of \\ Nuclear Medicine, The Tumor Hospital of \\ Yunnan Province, Kunming, 650II8, \\ People's Republic of China
}

Objective: This study aims to explore the correlation between the expression of mutant p53 protein and cellular atypia in early differentiated gastric adenocarcinoma (DGA).

Methods: A total of 107 cases of early DGA samples resected by endoscopic submucosal dissection (ESD) were collected from the Pathology Department of Beijing Friendship Hospital from January 2018 to December 2019. The EnVision two-step immunohistochemical method was used to detect the expression of mutant $\mathrm{p} 53$ protein in these cancer tissues, and the correlation with cell atypia was analyzed.

Results: In early DGA tissues, the expression rate of mutant p53 protein was significantly higher than in normal gastric mucosa $(P<0.01)$. However, the expression of mutant $\mathrm{p} 53$ protein was not correlated to age or gender $(P>0.05)$ but to the location of the tumor, depth of invasion, and degree of differentiation $(P<0.01)$. The expression of mutant p53 protein was closely correlated to cell atypia. Furthermore, this was weakly positive in low-grade atypical adenocarcinoma but strongly positive or negative in high-grade atypical adenocarcinoma, and there was a significant difference between these two $(P<0.01)$.

Conclusion: Mutant p53 protein is highly expressed in early DGA, which can be used as an auxiliary index for the diagnosis of early gastric cancer. The different expression patterns of mutant $\mathrm{p} 53$ protein in high-grade and low-grade atypical gastric cancers suggest that these may have different genetic changes.

Keywords: early gastric cancer, cell atypia, mutation, P53 protein

\section{Introduction}

Gastric cancer is one of the most common malignant tumors. The incidence and mortality of gastric cancer globally rank third and second, ${ }^{1}$ respectively. Early gastric cancer (EGC) means that the infiltration of cancer tissue is limited to the mucosa or submucosa, regardless of whether lymph node metastasis is present. The prognosis of gastric cancer is closely correlated to the tumor stage. Furthermore, the five-year survival rate of advanced gastric cancer patients is less than $50 \%$, and the five-year survival rate of EGC patients is $90 \%{ }^{2,3}$ Therefore, improving the detection rate of EGC is the key to improving the prognosis of gastric cancer patients. ${ }^{4}$ The mutation of the p53 gene plays an essential role in the occurrence and development of human tumors. Several studies have revealed that p53 gene mutations and the overexpression of mutant protein products can occur in all stages of tumor development. ${ }^{5}$ Therefore, the present study intends to evaluate the expression pattern of mutant p53 protein by observing its expression in early differentiated
Correspondence: Guang-Yong Chen Department of Pathology, Beijing Friendship Hospital, Capital Medical University, No. 95 of Yongan Street, Xicheng District, Beijing, 100050, People's Republic of China

Tel $+8610630144 I I$

Fax +861063016616

Email gycdr_sun06@163.com 
gastric adenocarcinoma (DGA) and its correlation with cell atypia to provide a basis for the occurrence, development, and pathological diagnosis of EGC.

\section{Materials and Methods}

\section{Subjects}

A total of 107 cases of early DGA samples were collected from the Pathology Department of Beijing Friendship Hospital from January 2018 to December 2019. Among these patients, 79 were male and 28 were female, and the ages ranged between 38-82 years old, with an average age of $63 \pm 8.6$ years old. Tumor location: The tumor was at the cardia in 22 patients, at the gastric fundus in 16 patients, at the gastric antrum junction in seven patients, at the gastric antrum in 49 patients, at the gastric angle in 11 patients, and at the pylorus in two patients. Tissue differentiation: 58 patients had highly differentiated adenocarcinoma, 31 patients had highly to moderately differentiated adenocarcinoma, and 18 patients had moderately differentiated adenocarcinoma. Cell atypia: 62 patients had low-grade tumors, and 45 patients had high-grade tumors. The infiltration depth was: T1a-LPM in 70 patients, T1aMM in 25 patients, and T1b-SM in 12 patients. This study was conducted in accordance with the Declaration of Helsinki and approved by the Ethics Committee of Beijing Friendship Hospital of China, Capital Medical University. Written informed consent was obtained from all participants.

\section{Methods}

The primary antibody and EnVision kits were all purchased from Fuzhou Maxim Biotechnology Co., Ltd. (China). The mutant p53 protein was stained using the EnVision method and observed under a light microscope.

\section{Result Interpretation Criteria}

The results of immunohistochemistry were interpreted according to the semi-quantitative sum-score method. Five areas were randomly selected for each section, and the expression in 1000 tumor cells was evaluated by high power microscopy $(400 \times)$. The double-blind interpretation was performed by two doctors with senior titles in the pathology department. The positive standard for $\mathrm{p} 53$ protein: Focusing on the nucleus, the scores for positive cells were as follows: $<5 \%$ of positively stained cells scored 0 points; 5-25\% scored 1 point, 26-50\% scored 2 points, $51-75 \%$ scored 3 points, and $>75 \%$ scored 4 points.
Staining intensity score: No staining scored 0 points, light yellow staining scored 1 point, brown-yellow staining scored 2 points, and tan staining scored 3 points. The final result was determined by adding the scores of the staining degree and staining intensity: $0-1$ points were regarded as $(-), 2-3$ points were regarded as weakly positive $(+), 4-5$ points were regarded as medium positive $(++)$, and 6-7 points were regarded as strongly positive $(+++)$, where $(+$ to +++ ) were all regarded as positive. ${ }^{6}$

\section{Statistical Analysis}

Data were statistically analyzed using statistical software SPSS 22.0. Count data were expressed in percentage (\%) and compared between groups using $X^{2}$-test and regression analysis. $P<0.05$ was considered statistically significant.

\section{Results}

\section{The Expression of Mutant p53 Protein in DGA and Paracancerous Tissue}

The expression of p53 protein was positive in 85 of 107 cases (79.4\%) with early DGA, among which 60 cases were weakly positive $(56.1 \%)$, one case was moderately positive $(0.9 \%)$, and 24 cases were strongly positive (22.4\%). Furthermore, compared to paracancerous tissues, the positive expression was relatively higher, and the difference was statistically significant $(P<0.01$, Table 1$)$. Mutant p53 protein was not expressed in paracancerous tissues (Figure 1A). The low and high expressions of mutant $\mathrm{P} 53$ protein in differentiated adenocarcinoma are shown in Figure 1B and 1C

\section{The Correlation Between the Positive Expression of Mutant p53 Protein and the Clinicopathological Characteristics of Early DGA}

The positive expression of mutant p53 protein was correlated with the degree of tumor differentiation, location of the

Table I The Expression of Mutant p53 Protein in DGA and Paracancerous Tissue

\begin{tabular}{|l|l|l|l|l|l|l|}
\hline Group & $\mathbf{n}$ & + & - & $\begin{array}{l}\text { Positive } \\
\text { Rate (\%) }\end{array}$ & $\chi^{2}$ & P value \\
\hline DGA & 107 & 85 & 22 & 79.4 & 141.008 & 0.000 \\
\hline $\begin{array}{l}\text { Paracancerous } \\
\text { tissues }\end{array}$ & 107 & 0 & 107 & 0 & & \\
\hline
\end{tabular}

Abbreviation: DGA, differentiated gastric adenocarcinoma. 
A

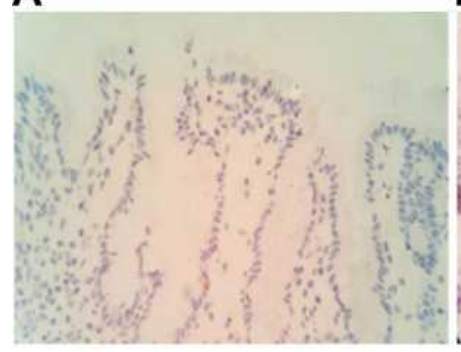

B

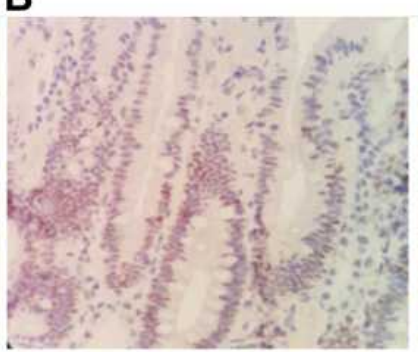

C

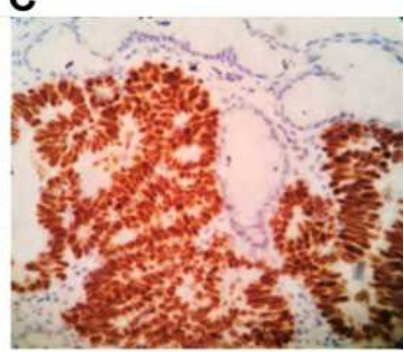

Figure I Immunohistochemical staining to observe the expression of mutant p53 protein in differentiated gastric adenocarcinoma and adjacent tissues (400x). (A) Mutant p53 protein was not expressed in paracancerous tissues. (B) The low expression of mutant P53 protein in differentiated adenocarcinoma. (C) The high expression of mutant P53 protein in differentiated adenocarcinoma.

tumor, and depth of invasion $(P<0.01)$. With the decrease in the degree of tumor differentiation, the positive rate of mutant p53 protein expression also decreased. The positive rate of mutant $\mathrm{p} 53$ protein expression was the highest at the gastric antrum of adenocarcinoma patients and lowest at the gastric cardia. Furthermore, the expression rate of mutant p53 protein was significantly higher in T1a than in T1b. However, the positive expression of mutant p53 protein was not correlated to age and gender $(P>0.05$, Table 2$)$.

\section{The Correlation Between the Expression of Mutant p53 Protein and Cell Atypia}

Among the 62 cases of low-grade atypical adenocarcinoma tissues, the expression of mutant p53 protein was negative in one case $(1.61 \%)$, weakly positive in 60 cases $(96.77 \%)$, moderately positive in zero cases $(0 \%)$, and strongly positive in one case $(1.61 \%)$. Among the 45 cases of high-grade atypical adenocarcinoma tissues, the expression of mutant p53 protein was negative in 21 cases $(46.67 \%)$, weakly positive in zero cases $(0 \%)$, moderately positive in one case $(2.22 \%)$, and strongly positive in 23 cases $(51.11 \%)$. Figure $2 \mathrm{~A}$ and D show the morphological characteristics of low-grade atypical adenocarcinoma and the low expression pattern of mutant P53 protein. Figure $2 \mathrm{~B}$ and $\mathrm{E}$ show the morphological characteristics of highgrade atypical adenocarcinoma and the high expression pattern of mutant P53 protein. Figure 2C and F show the morphological characteristics of high-grade atypical adenocarcinoma and the negative expression pattern of mutant P53 protein. Two sets of figures of $2 \mathrm{~B} / 2 \mathrm{E}$ and $2 \mathrm{C} / 2 \mathrm{~F}$ show two expression patterns of mutant P53 protein in highgrade atypical adenocarcinoma.

The difference between the expression of mutant p53 protein and cell atypia was statistically significant $(P<$ 0.01 , Table 3 ). The regression analysis results revealed that the expression of mutant p53 protein was positively correlated with cell atypia. With the increase in nuclear grade, the expression of mutant p53 protein was enhanced, and there was a significant correlation between these two $(P<0.01)$. The regression equation was as follows: $\mathrm{y}^{\wedge}=1.417+0.406$ nuclear grade.

Table 2 The Correlation Between the Positive Expression of Mutant p53 Protein and the Clinicopathological Characteristics of Early DGA

\begin{tabular}{|c|c|c|c|c|}
\hline \multirow{2}{*}{$\begin{array}{l}\text { Clinicopathological } \\
\text { Characteristics }\end{array}$} & \multirow[t]{2}{*}{$\mathbf{n}$} & \multicolumn{3}{|c|}{ p53 Protein } \\
\hline & & $\begin{array}{c}\text { Positive } \\
(n, \%)\end{array}$ & $\chi^{2}$ & $P$ value \\
\hline \multicolumn{5}{|l|}{ Age (year) } \\
\hline$<60$ & 33 & 29 (87.9\%) & 2.081 & 0.149 \\
\hline$\geq 60$ & 74 & $56(75.7 \%)$ & & \\
\hline \multicolumn{5}{|l|}{ Gender } \\
\hline Male & 79 & 63 (79.7\%) & 0.484 & 0.486 \\
\hline Female & 28 & $24(85.7 \%)$ & & \\
\hline \multicolumn{5}{|l|}{ Differentiated degree } \\
\hline High differentiation & 58 & 52 (98.7\%) & 10.523 & 0.005 \\
\hline High to medium & 31 & $25(80.6 \%)$ & & \\
\hline \multicolumn{5}{|l|}{ differentiation } \\
\hline Middle differentiation & 18 & $10(55.6 \%)$ & & \\
\hline \multicolumn{5}{|l|}{ Location of tumor } \\
\hline Gastric cardiac part & 22 & II (50.0\%) & 17.86 & 0.001 \\
\hline Gastric fundus & 16 & I4 (87.5\%) & & \\
\hline Gastric antrum & 49 & 45 (91.8\%) & & \\
\hline Gastric angle & II & $9(81.8 \%)$ & & \\
\hline Other & 9 & $6(66.7 \%)$ & & \\
\hline \multicolumn{5}{|l|}{ Depth of invasion } \\
\hline Tla & 95 & $80(84.2 \%)$ & 11.806 & 0.001 \\
\hline TIb & 12 & $5(41.7 \%)$ & & \\
\hline
\end{tabular}

Abbreviation: DGA, differentiated gastric adenocarcinoma. 
A

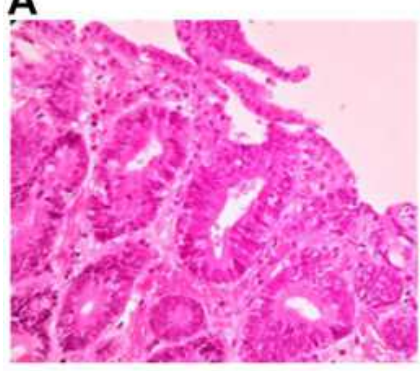

D

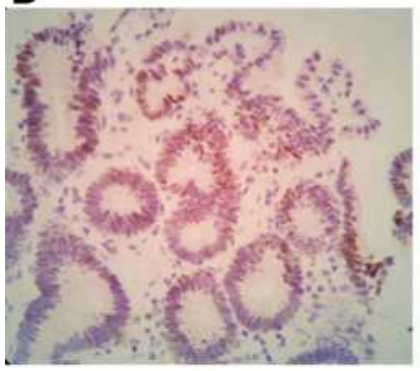

B

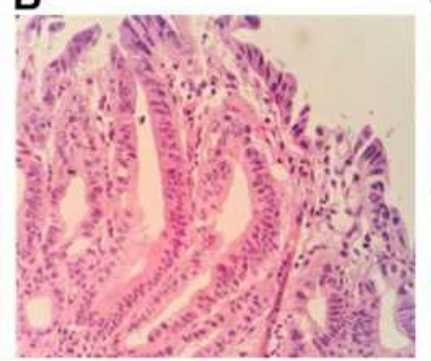

E

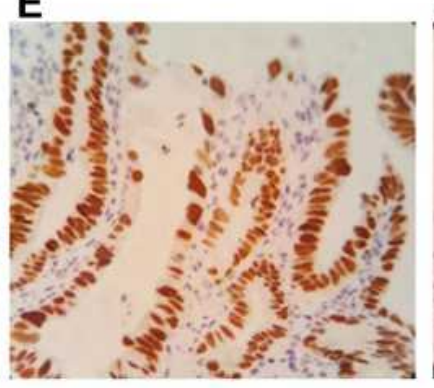

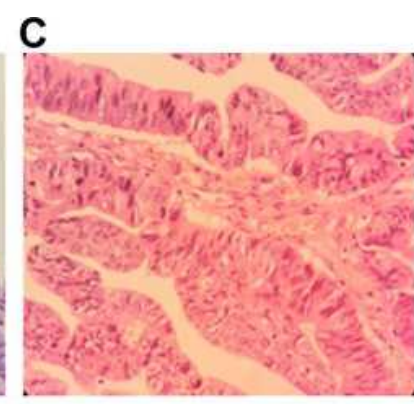

$\mathbf{F}$

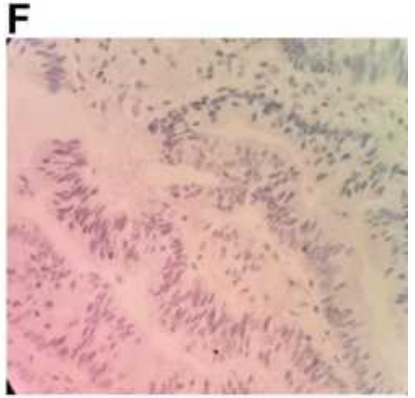

Figure 2 Immunohistochemical observation of the expression of mutant p53 protein in different cell atypical adenocarcinomas (400x). (A) Low-grade atypical adenocarcinoma (HE). (B) High-grade atypical adenocarcinoma (HE). (C) High-grade atypical adenocarcinoma (HE). (D) The low expression of mutant p53 protein in lowgrade atypical adenocarcinoma. (E) The high expression of mutant $\mathrm{p} 53$ protein in high-grade atypical adenocarcinoma. (F) No expression of mutant $\mathrm{p} 53$ protein in high-grade atypical adenocarcinoma.

\section{Discussion}

In recent years, the detection rate of EGC was $19.5 \%$ in all gastric cancers in China. ${ }^{7}$ Although this is increasing yearly, this remained significantly lower by $50-80 \%$ compared with Japan and South Korea. Therefore, further improving the detection rate of EGC is of great significance for prolonging patients' overall survival and quality of life. At present, the diagnosis of EGC mainly depends on gastroscopy screening combined with pathological biopsy. The difficulty of the pathological diagnosis of biopsy of EGC lies in the limited sample size and atypical lesions, especially in the differentiation between cancer and regenerative disease. The accuracy of pathological diagnosis would be directly correlated to the further treatment of patients.

The p53 gene is a tumor suppressor gene located on chromosome 17, which encodes the p53 protein. This can be divided into wild-type and mutant-type, according to whether or not it is mutated. Wild-type p53 protein can repair damaged cells and maintain the stability of genomes, ${ }^{8}$ but its half-life is short, its content in cells is low, and it is not generally expressed, while mutant p53 protein loses the ability to repair cells and promotes the development of tumors.

One of the results of the present study revealed that the positive rate of mutant p53 protein was $79.4 \%$ in early DGA, and the result was higher than those reported by Kang and Lin et al. ${ }^{9,10}$ The positive rate of mutant p53 protein was $0 \%$ in paracancerous tissues, and the difference between these two was statistically significant $(P<$ $0.01)$. Therefore, the detection of mutant p53 protein would be helpful for the pathological diagnosis of EGC. In addition, the reason that the positive rate of mutant $\mathrm{p} 53$ protein in the present study was significantly higher than that in other reports may be correlated to the degree of tumor differentiation and stage.

Table 3 The Correlation Between the Expression of Mutant p53 Protein and Cell Atypia

\begin{tabular}{|c|c|c|c|c|c|c|c|c|c|c|c|c|c|}
\hline \multirow[t]{2}{*}{ Nuclear Atypical } & \multirow[t]{2}{*}{$\mathbf{n}$} & \multicolumn{12}{|c|}{ P53 } \\
\hline & & - & $\chi^{2}$ & $P$ value & + & $\chi^{2}$ & $P$ value & ++ & $\chi^{2}$ & $P$ value & +++ & $\chi^{2}$ & $P$ value \\
\hline Low-grade & 62 & I & \multirow[t]{2}{*}{32.405} & \multirow[t]{2}{*}{0.000} & 60 & \multirow[t]{2}{*}{99.142} & \multirow[t]{2}{*}{0.000} & 0 & \multirow[t]{2}{*}{1.346} & \multirow[t]{2}{*}{0.246} & I & \multirow[t]{2}{*}{36.718} & \multirow[t]{2}{*}{0.000} \\
\hline High-grade & 45 & 21 & & & 0 & & & I & & & 23 & & \\
\hline
\end{tabular}


In the Convention on the Treatment of Gastric Cancer, ${ }^{11}$ according to the degree of formation of the gland duct structure, DGA can be divided into two types: tubular adenocarcinoma (highly differentiated and moderately differentiated) and papillary adenocarcinoma. All specimens in the present study were tubular adenocarcinoma. According to the degree of cell atypia, this can be divided into low-grade atypical adenocarcinoma and highgrade atypical adenocarcinoma. ${ }^{12}$ The diagnostic criteria are as follows: the former has a shuttle nucleus, the cells are arranged on the basal side, there is basic nuclear pole orientation, and the nucleus-to-cytoplasm ratio is $<50 \%$; the nucleus of the latter is quasi-round, it loses polarity, and the nucleus-to-cytoplasm ratio is $>50 \%$. In the present study, 62 cases had low-grade atypical adenocarcinoma, while 45 cases had high-grade atypical adenocarcinoma.

The second result of the present study revealed that the expression of mutant p53 protein was weakly positive in low-grade atypical adenocarcinoma but primarily strongly positive or negative in high-grade atypical adenocarcinoma, and the difference between these two was statistically significant $(P<0.01)$. This result suggests that the atypia of cells is correlated to the mutation of the p53 gene. The correlation between these two was further investigated by regression analysis, and the results revealed that the expression of mutant p53 protein was positively correlated with cell atypia. In the present study, the regression equation between nuclear grade and mutant p53 protein expression was further analyzed to provide a new basis and auxiliary method for diagnosing atypical adenocarcinoma. In addition, the present study also revealed that the expression of mutant p53 protein was correlated to the degree of tumor differentiation and depth of invasion but was not correlated with age or gender. This is partly inconsistent with the conclusion described by Waraya et $\mathrm{al},{ }^{13}$ in which the expression of mutant $\mathrm{p} 53$ protein was correlated with age, gender, and degree of tumor differentiation. Further studies and comparisons are needed to determine whether this is correlated to the fact that the samples selected in the present study were all early differentiated adenocarcinoma tissues.

The occurrence and development of gastric cancer is a process of qualitative change caused by multiple factors. Its pathogenesis involves the genetic and epigenetic changes of many genes and molecular signaling pathways. To date, the etiology and pathogenesis of gastric cancer have not been fully elucidated. In 2002, Singer et $\mathrm{al}^{14}$ proposed a dualistic model of serous ovarian carcinoma.
On this basis, in 2004 , Malpica et $\mathrm{al}^{15}$ proposed a twolevel grading system of histopathology based on the atypia of tumor nuclei, wherein serous ovarian carcinoma was divided into low-grade serous carcinoma and high-grade serous carcinoma. There were different tumorigenesis pathways between these two, in which the former was mainly caused by KRAS or BRAF gene mutation, and the p53 gene was mostly wild-type, while the latter was caused primarily by $\mathrm{p} 53$ gene mutation, and the KRAS or BRAF gene is mostly wild-type.

Furthermore, the clinicopathological characteristics, treatment, and prognosis of these two differed. In the present study, the expression pattern of $\mathrm{p} 53$ protein in early DGA was similar to that in serous ovarian carcinoma. From this, the investigators speculated that there are similar dualism models in DGA. There are different tumorigenesis pathways between high-grade atypical adenocarcinoma and low-grade atypical adenocarcinoma, and the occurrence of high-grade atypical adenocarcinoma is mainly correlated to p53 gene mutation. Indeed, this needs to be proven through further studies.

The results of the present study revealed that in early DGA tissues, mutant p53 protein was highly expressed and significantly correlated to the cell atypia. This can be used as an auxiliary index in the diagnosis of early gastric cancer. The different expression patterns of mutant p53 protein in high-grade and low-grade atypical gastric cancers suggest that these may have different genetic changes. In the future, the investigators would further expand the number of specimens and investigate the differences between these two indepth to provide a new basis for the pathogenesis and pathological diagnosis of gastric cancer.

There were some limitations in the study. First, the number of cases in the present study was limited. Second, all cases in the present study were tubular adenocarcinoma without papillary adenocarcinoma. Third, it was difficult to determine the true expression of mutant p53 protein merely by immunohistochemistry. Therefore, the nucleic acid detection method should be further used for verification.

\section{Conclusion}

In early DGA tissues, mutant p53 protein is highly expressed. Furthermore, its expression level is also significantly correlated with the pathological characteristics and cell atypia of early DGA. This expression level can be used as an auxiliary index in the diagnosis of early gastric cancer. The different expression patterns of mutant p53 
protein in high-grade and low-grade atypical gastric cancers suggest that these may have different genetic changes.

\section{Disclosure}

The authors report no conflicts of interest in this work.

\section{References}

1. Torre LA, Bray F, Siegel RL, Ferlay J, Lortet-Tieulent J, Jemal A. Global cancer statistics, 2012. CA Cancer J Clin. 2015;65(2):87-108. doi:10.3322/caac. 21262

2. Saragoni L. Upgrading the definition of early gastric cancer: better staging means more appropriate treatment. Cancer Biol Med. 2015;12 (4):355-361. doi:10.7497/j.issn.2095-3941.2015.0054

3. Leung WK, Wu MS, Kakugawa Y, et al.; Asia Pacific Working Group on Gastric Cancer. Screening for gastric cancer in Asia: current evidence and practice. Lancet Oncol. 2008;9(3):279-287. doi:10.1016/ S1470-2045(08)70072-X

4. Leja M, You W, Camargo MC, Saito H. Implementation of gastric cancer screening - the global experience. Best Pract Res Clin Gastroenterol. 2014;28(6):1093-1106. doi:10.1016/j.bpg.2014.09.005

5. Prass K, Scharff A, Ruscher K, et al. Hypoxia-induced stroke tolerance in the mouse is mediated by erythro-poietin. Stroke. 2003;34 (8):1981-1986. doi:10.1161/01.STR.0000080381.76409.B2

6. Zhao ZH, Tian Y, Chen KS. Expression of c-myc and MDM2 proteins in multiple myeloma and their clinical significance. J Clin Exp Pathol. 2017;33(2):165-168.
7. Chinese federation of gastrointestinal cancer surgery. Data report of Chinese federation of gastrointestinal cancer surgery (2014-2016). Chin J Pract Surg. 2018;38(1):90-93.

8. Qin Y, Liang LP, Zheng XZ, et al. Value of detection of DNA mismatch repair proteins deficiency by immunohistochemistry in predicting tumor microsatellite status. Zhonghua Bing Li Xue Za Zhi. 2015;44(10):704-708.

9. Kang X, Song HX, Guo QN. Expression of IBP and p53 in gastric adenocarcinoma and its clinical significance. Acta Acad Med. 2019;41(03):219-226.

10. Lin DD, Zhang YQ. Expression changes of P53 and ki-67 proteins in gastric cancer tissues and their significance. Shandong Med J. 2015;55(45):48-49.

11. Gastric and intestinal editorial board. Gastric and Intestinal Classification of Gastric Diseases to Be Mastered. Shenyang: Liaoning science and technology press; 2017:16-18.

12. Watanabe $\mathrm{H}$. What is the malignancy diagnosis of gastric cancer and colorectal cancer. Pathol Clin. 2005;23(9):932-945.

13. Waraya M, Yamashita K, Ema A, Katada N, Kikuchi S, Watanabe M. Exclusive association of p53 mutation with super-high methylation of tumor suppressor genes in the p53 pathway in a unique gastric cancer phenotype. PLoS One. 2015;10(10):e0139902. doi:10.1371/journal. pone.0139902

14. Singer G, Kurman RJ, Chang HW, Cho SK, Shih I. Diverse tumorigenic pathways in ovarian serous carcinoma. Am J Pathol. 2002;160 (4):1223-1228. doi:10.1016/S0002-9440(10)62549-7

15. Malpica A, Deavers MT, Lu K, et al. Grading ovarian serous carcinoma using a two-tier system. Am $J$ Surg Pathol. 2004;28 (4):496-504. doi:10.1097/00000478-200404000-00009

\section{Publish your work in this journal}

Cancer Management and Research is an international, peer-reviewed open access journal focusing on cancer research and the optimal use of preventative and integrated treatment interventions to achieve improved outcomes, enhanced survival and quality of life for the cancer patient.
The manuscript management system is completely online and includes a very quick and fair peer-review system, which is all easy to use. Visit http://www.dovepress.com/testimonials.php to read real quotes from published authors. 